\title{
A Bench Measurement of the Energy Loss of a Stored Beam to a Cavity
}




\section{A Bench Measurement of the Energy Loss}

of a Stored Beam to a Cavity

\section{Contents}

$\begin{array}{llr}\text { 1. } & \frac{\text { Page }}{2} \\ \text { 2. } & \text { Introduction } & 3 \\ 3 . & \text { Principle of the Method } & 10 \\ \text { 4. } & \text { Experimental Set-Up } & 12 \\ 5 . & \text { Input Pulse } & 15 \\ 6 . & \text { Oscillograph } & 15 \\ \text { 7. } & \text { Interpretation of the Data } & 20 \\ \text { 8. } & \text { Expected Signals } \\ \text { 9. } & \text { Center Conductor } & 24 \\ \text { 10. } & \text { Synthetic Pulse Method } & 27 \\ & \text { Conclusions } & 31 \\ & \text { Acknowledgement }\end{array}$

\section{Summary}

A rather simple electronic bench experiment is proposed for obtaining a measure of the impulse energy loss of a stored particle bunch to an rf cavity or other vacuum-chamber structure -the so-called "cavity radiation". The proposed method is analysed in some detail. 


\section{Introduction}

Theoretical estimates of the loss of energy from the beam of a highcurrent electron storage ring due to the transient excitation of the rf accelerating cavities indicate that the effect may make significant demands for increased rf power, and may have other deleterious effects as we11.1 Unfortunately, theoretical estimates can be made only for idealized cavities whose shapes are very different from the actual cavities. Also, the theories, themselves, use approximations whose consequences are uncertain. In view of the theoretical uncertainties, it would be highly desirable -- and of some practical importance in the design of a large storage ring such as PEP -- to have some reliable determination of the energy loss that will occur in the ring. It is contemplated that the loss will be measured for the cavities of SPEAR-2, but such measurements -- particularly if extended to other cavity-like structures of the vacuum system -- may be difficult, and will certainly be time-consuming and expensive.

I would like to propose that the transient energy loss of circulating bunch in a storage ring as it traverses a cavity can be determined by an electronic bench measurement. I consider in this note what the set-up might look like for a measurement on the PEP cavities. The method would also be useful for determining the energy loss of a bunch in traversing various parasitic cavities -- vacuum boxes and the like -- in the PEP storage ring. 


\section{Principle of the Method}

When a stored particle bunch traverses a cavity, the electrical charge and current of the bunch produce electric and magnetic fields at the walls of the vacuum chamber. These fields generate what we may call "induced" charges and currents in the walls, which in turn produce "secondary" fields that can appear at the circulating bunch and can give rise to deflections or changes of energy of the particles in the bunch. (These secondary fields have at times been referred to as "wake" fields.) I am, here, interested only in the longitudinal electric fields that will subtract energy from the stored bunch. Of particular concern at the moment are the secondary fields that arise from any irregularity in the storage ring vacuum chamber -- and, in particular, such gross irregularities as the $r f$ accelerating cavities. The method I am proposing is intended to give a measure of the energy loss of a bunch due to such irregularities.

Imagine that an appropriate section of the vacuum chamber of PEP, including, say, an rf cavity, is fitted with a smal1 center conductor so as to make an irregular coaxial transmission line. The idea is shown schematically in Fig. 1. Next, imagine that a short pulse of current is introduced on the left end of the center conductor. The time-dependence of the current in the pulse is intended to produce the same Gaussianshaped time variation that occurs in a stored electron bunch. The current pulse will travel along the center conductor with the speed c, and will have an instantaneous charge density that corresponds closely to the charge density in a travelling bunch. And so long as this "model bunch" is travelling along the uniform pipe, it will produce fields in the pipe nearly 

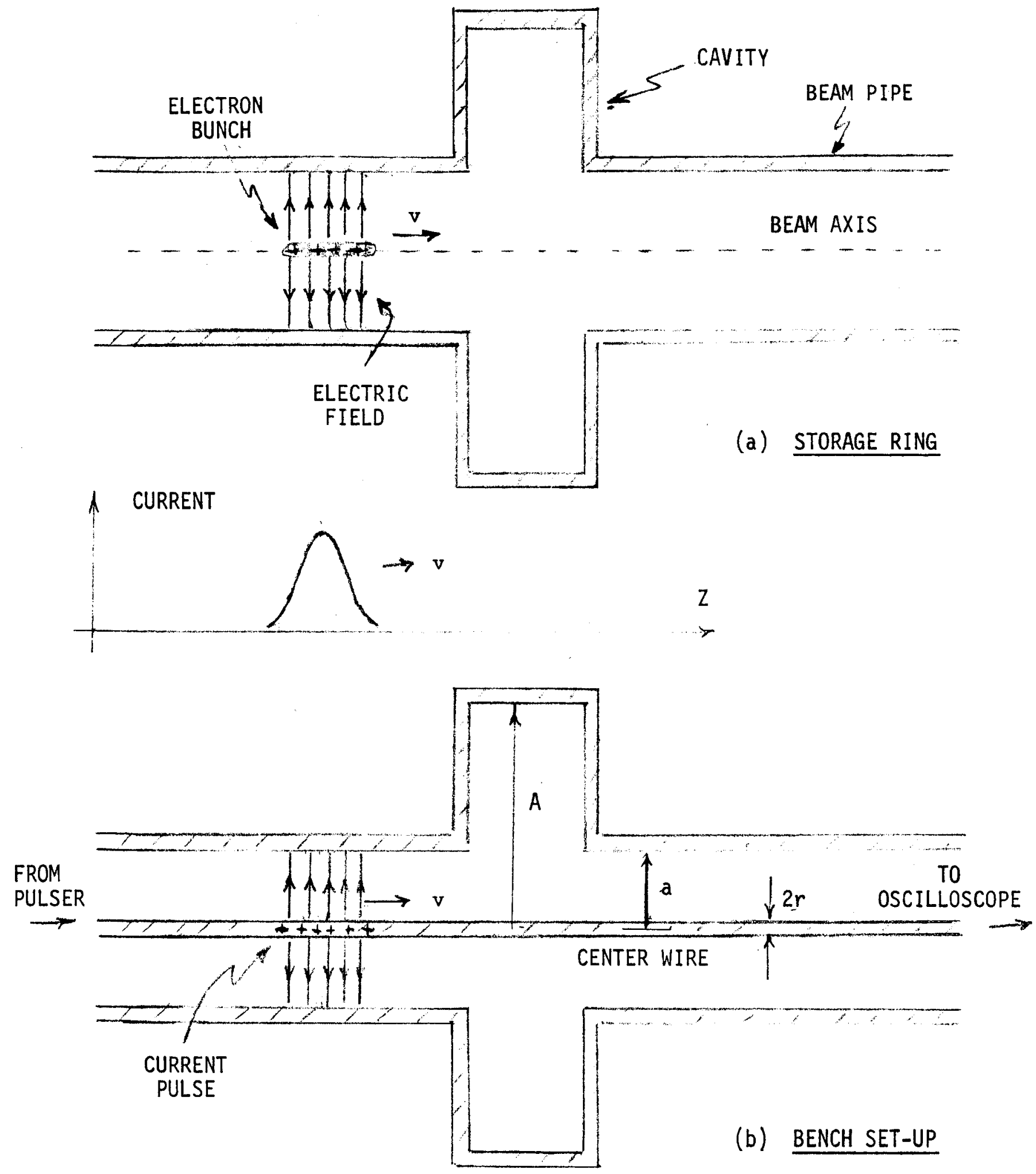

Fig. 1. Diagram of Bench Set-Up 
identical to the fields produced by a bunch of ultra-relativistic electrons. (The differences are, small so long as the bunch length $\ell$ is much larger than $a / \gamma$, where $a$ is the radius of the chamber, and $\gamma=E / m c^{2}$ of the particles.) Under these circumstances, it is well known, the secondary fields at the bunch (or at the pulse on the wire) are small and proportional to the resistivity of the walls. I am going to assume here that the walls have a sufficiently low resistivity that these fields can be neglected. What happens when the stored bunch or current pulse arrives at the cavity? The induced currents and charges on the walls are changed and new secondary fields are produced. The longitudinal electric fields that occur at the bunch will change the energy of the particles in the bunch, but will not -- assuming ultra-relativistic particles -- have any significant effect on the charge and current distribution of the bunch. The net change of the energy of each particle is the integral of the secondary electric field along its path, and the total energy lost by the bunch is the sum of the energy changes of all of the particles.

Assume for a moment that the current pulse on the wire is not disturbed as it goes through the cavity. Then its current and charge remain the same as those of the bunch, and, I would maintain, it follows that the charges induced in the walls by the pulse, as well as the secondary fields generated, are identical in the two cases. So the energy removed from the pulse by the secondary fields is the same as from the bunch. If we can measure the energy removed from the coaxial current pulse by the secondary fields, we will have a measure of the energy loss of a bunch while traversing the same structure.

In reality, there are, of course, differences. For one thing, the 
current pulse will not remain unchanged as it goes through the cavity. The secondary fields at the center conductor will produce "secondary" currents that will combine with the initial pulse to produce a modified pulse. The magnitude of the secondary current depends, however, on the diameter of the center conductor, and can, in principle, be made as small as we wish by the use of a sufficiently small central wire. Let's assume, for example, that we can make the disturbance to the original pulse, say, ten or twenty percent of the original pulse amplitude.

There is another important way in which the bench set-up differs from the storage ring. The secondary currents in the bench set-up will produce new "secondary" induced charges in the chamber walls that will in turn give rise to "tertiary" currents in the center wire, and so on ad infinitum. I would like to argue that these "higher-order" effects do not change significantly the energy loss from the initial pulse. First, they can be made sma11. Second, these effects will all occur with time delays (due to the back-and-forth transit of the electromagnetic fields) so that they wi11, in general, produce additional fields and currents on the wire that occur well behind the initial pulse and can therefore be distinguished from the secondary fields that we wish to observe. (This statement is, I think, particularly true when the bunch length is less than or comparable to the diameter of the vacuum pipe -- which is also the situation of practical interest to us.

The most striking difference between the two situations is what happens to the electromagnetic energy left behind in the chamber after the passage of the beam bunch or current pulse. In the absence of the center wire, the energy appears in the oscillations of a large number of normal 
modes of the vacuum chamber-cavity system, and is transformed eventually into heat energy in the walls as the oscillating fields decay. With the center wire present, two changes occur. First, the characteristics of the normal modes (for example, frequency and field distribution) are changed. And, second, the energy of the modes is, to a large extent, coupled into the center wire and travels along it to be dissipated in the terminations at the two ends. It might appear that these differences are so great as to make the two situations quite disparate. My intuition, however, would say that (1) if the frequencies only of the normal modes are not grossly modified, and (2) if the duration of the beam pulse is short in comparison with the relaxation times of the residual fields with the wire present, then the energy removed from the pulse -- and left behind as field energy in the chamber -- will be similar to the energy removed from the beam bunch.

Suppose we try to imagine what would be the fields produced in a cavity by the transit of a beam bunch. My guess would be that the fields in the cavity immediately after the passage of the bunch might look as sketched in Fig. 2. And -- I see no reason to think that the field distribution would be changed significantly by the presence of a small wire along the axis. What will be different, certainly, is the behavior after the pulse has passed -- as the stored fields rattle around searching for an escape. But this later comportment has little to do with the energy contained initially in the fields left behind by the transit of the current pulse.

According to estimates I have made, both of the two conditions mentioned above for the validity of the method will apply in the circumstances 


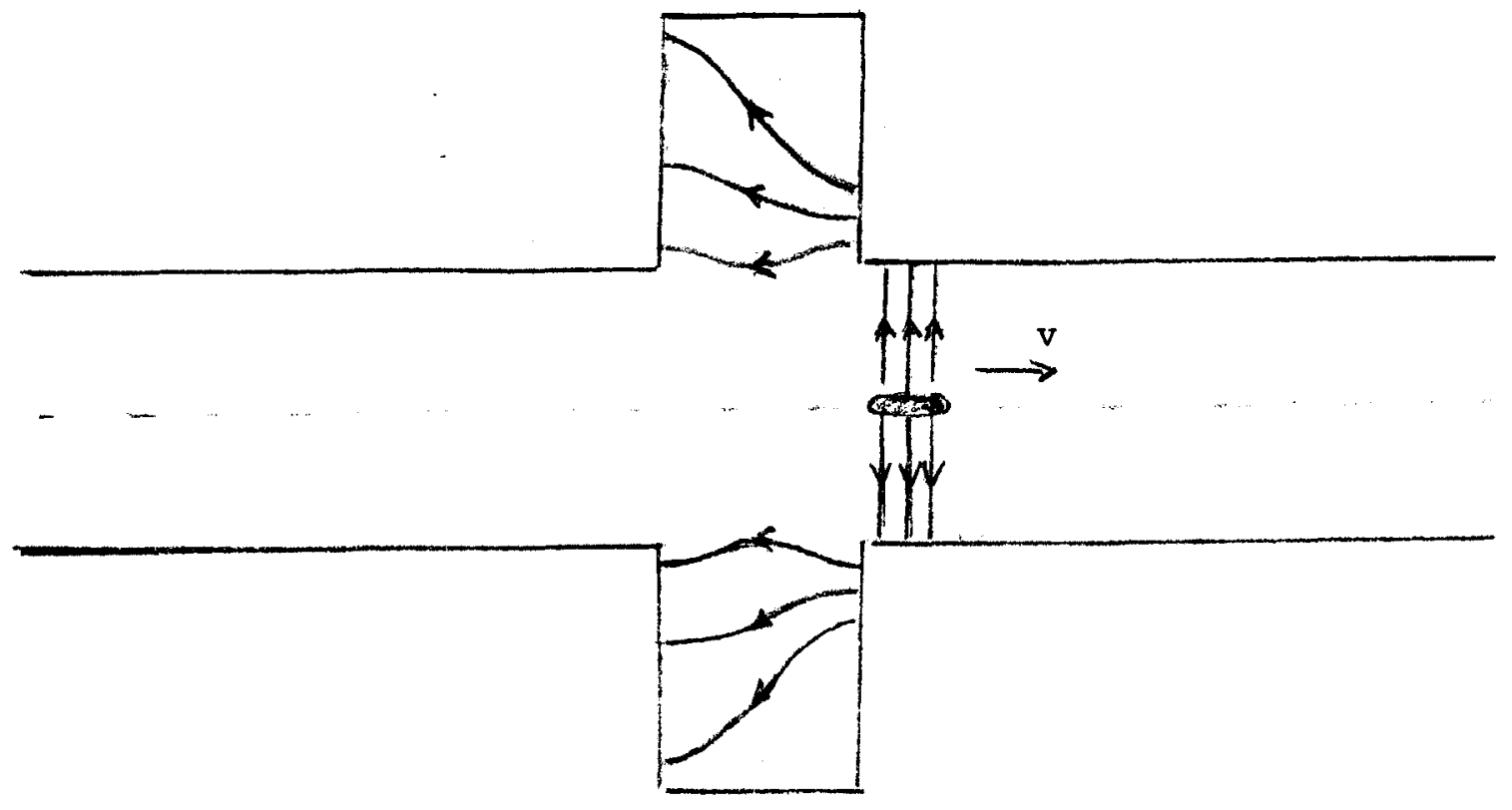

Fig. 2. Conjectured Distribution of the Electric

Field Just After a Bunch Has Traversed

a Cavity 
contemplated for measurements related to the PEP cavities. For example, I estimate that a 2-millimeter wire placed along the axis of a PEP cavity and terminated in a resistance appropriate to the transmission line it makes with the vacuum chamber will change the fundamental frequency of $360 \mathrm{MHz}$ by about $10 \%$ and reduce the quality factor $Q$ of that mode to about 8 . The fundamental mode will discharge most of its energy down the wire in a time $2 Q / \omega_{0}$, which is about $7 \times 10^{-9} \mathrm{sec}$, a time long compared with the pulse duration of $0.3 \times 10^{-9} \mathrm{sec}$. The same should be true of most of the other significant modes. The details of these estimates are given in Appendix A.

My confidence in these estimates was bolstered by a simple experiment. A 3/32-inch-diameter copper rod was mounted on insulators so that it extended along the axis of a 540-MHz cavity intended for use in SPEAR, and the resonant frequency of the fundamental mode was measured. I had estimated roughly that the frequency would be increased over the frequency without the rod by about $20 \%$. The observed change was $22 \%$. Also, a qualitative exploration of the electric field distribution showed that it was as I expected.

The experimental technique I am proposing is, then, based on the following ideas: That a current pulse that simulates approximately a travelling electron bunch can be sent along a small wire inserted on the axis of a storage ring vacuum pipe. That the secondary reaction fields produced by the irregularities of the chamber wall will be approximately the same for the current pulse as for the travelling bunch. That a measurement of the energy loss by the current pulse in traversing an irregular segment of beam chamber is a measure of the energy loss by a beam bunch in traversing the same segment. That the differences in the two situations can be made 
smal1, and can be -- at least in part -- corrected for.

In the rest of this note, I will put forth some of the quantitative aspects of a bench set-up that might be realized in practice.

\section{Experimental Set-Up}

A schematic diagram of the proposed experimental set-up is shown in Fig. 3. A fast-pulse generator is fed through a pulse-shaping network and coupling to a tapered matching section to the coaxial transmission line formed by the first section of beam chamber -- perhaps 1 meter long -and the central wire. After the cavity, there is a second section of beam chamber and an output tapered section which couples the output pulse to the detector head of a sampling oscillograph. Because of the rather large diameter of the beam chamber and the small diameter of the central wire, the coaxial system will have many possible modes of transmission for the current pulse. It will be necessary to take care that the pulser and matching section launch a current pulse of appropriate time dependence and in the appropriate transverse-field mode. Also it is necessary that the end coupling devices provide a proper termination for the signals that reach them.

For the interpretation of the data, it is necessary to have the record of an "original pulse" -- the pulse that would be transmitted through the system in the absence of a cavity. To obtain this original signal, it would be useful to make the cavity demountable, so that it can be replaced by a section of beam chamber -- making a continuous coaxial line. Alternatively, the cavity could be fitted with a sliding sleeve that could either cover up the cavity or expose it. An over-all check of the performance of 
-- 11 --

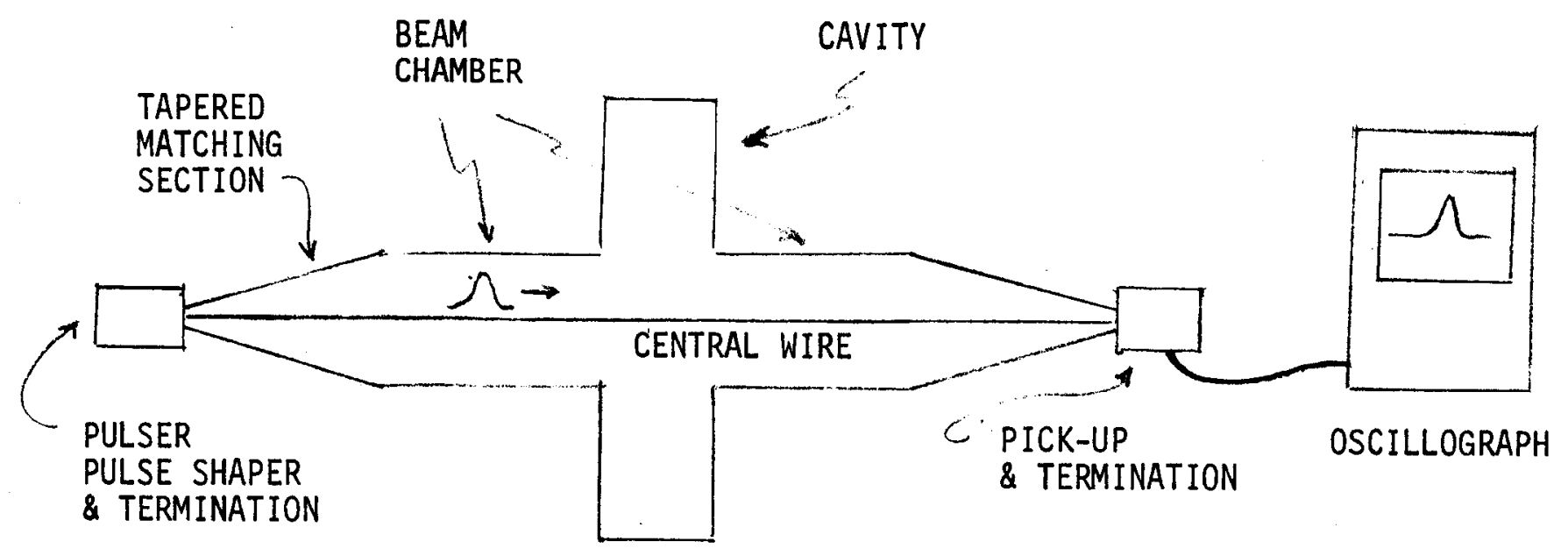

Fig. 3. Schematic Diagram of the Proposed

Experiment 
the pulser and terminations will, of course, be obtained from the appearance of the recorded output of the original pulse. The basic measurement of the method consists of obtaining a recording of the original pulse (sans cavity) and then a recording of the pulse modified by the presence of the cavity. The analysis of these records requires obtaining the difference between these two signals -- which may be perhaps $5 \%$ to $20 \%$ of the original pulse -- so some care will be required to maintain a good reproducibility of the measurements -- hopefully, with a precision of about one percent or better. This is the accuracy that is required if the final answer is to have an error no larger than $10 \%$ to $20 \%$, which would be nice. I am told by those experienced with fast pulses that the desired reproducibility can be achieved.

\section{Input PuTse}

The circulating bunch in a storage ring has a current distribution in time (at a fixed place) that is expected to be nearly "Gaussian"; that is, distributed according to

$$
i_{0}(t)=I_{0} e^{-2 t^{2} / \tau^{2}},
$$

where $\tau$, the nominal bunch duration, is taken to be twice the $r m s$ spread. The total charge in the pulse is

$$
q_{0}=\int i(t) d t=\sqrt{\frac{2}{\pi}} I_{0} \tau \text {. }
$$

In the bench test, we should probably try, to the extent possible, to use 
a current pulse with the same dependence on time, although, as will be discussed in Section 9, that is not strictly necessary -- and may, in fact, not be desirable.

At low currents the bunch length $\underline{\ell}$ in PEP will be about $5 \mathrm{~cm}$. At high stored-beam intensities, however, the bunch may be much longer -perhaps as long as $15 \mathrm{~cm}$-- if PEP has an anomalous bunch lengthening like that observed in most rings. It would be useful to make energy loss measurements with, say, three pulse lengths: $5 \mathrm{~cm}, 10 \mathrm{~cm}$ and $15 \mathrm{~cm}$. In the following discussion I shal1 take $10 \mathrm{~cm}$ as an illustrative bunch length. The bunch duration $\tau$ is, of course, related to the bunch length $\ell$ by

$$
\tau=\frac{\ell}{c}=\frac{2 \sigma_{y}}{c} \text {, }
$$

where $\sigma_{y}$ is the standard deviation of the longitudinal spread. We are interested in pulse duration $\tau$ of about 330 picoseconds. It will be convenient to refer to $\tau$ also as the pulse "length" -- meaning, of course, "length in time".

Pulsers are available with pulse lengths as small as 35 picoseconds or so. Perhaps a pulser can be found that gives a good approximation to a Gaussian shape with a length of 330 picoseconds. Otherwise it may be necessary to lengthen and shape a shorter pulse by passing it through a "low-pass" network. There is a general theorem that the impulse response of a chain of low-pass filters approaches, with increasing number of stages, a Gaussian shape, so it should not be too difficult to generate a pulse which gives a suitable approximation to a stored bunch.

It will be useful to have some idea about the typical frequency 
components in the Fourier spectrum of the pulse. The pulse of Eq. (1) has a distribution of Fourier amplitudes given by

$$
A(\omega)=A_{0} e^{-\omega^{2} / 2 \omega_{0}^{2}},
$$

with

$$
\omega_{0}=\frac{2}{\tau} \text {; or } f_{0}=\frac{\omega_{0}}{2 \pi}=\frac{1}{\pi \tau} \text {. }
$$

I shal1 take $\omega_{0}$ (or $t_{0}$ ) as the "typical" frequency in the pulse. The "half-power" frequency $f_{\frac{1}{2}}$ is related to $\tau$ by

$$
f_{\frac{1}{2}}=\frac{\sqrt{\ln 2}}{2 \pi} \omega_{0}=\frac{\sqrt{\ln 2}}{\pi \tau}=\frac{0.265}{\tau} \text {. }
$$

For a $10-\mathrm{cm}$ pulse $\left(\tau=330\right.$ picoseconds), $\omega_{0}=6.1 \mathrm{GHz}$ and $f_{\frac{1}{2}}=0.8 \mathrm{GHz}$. The various components of the set-up -- connectors, tapered lines and such -- will need to deal faithfully with frequencies well above $1 \mathrm{GHz}$. The appearance of the original pulse (no cavity) will, of course, give some measure of this fidelity. It must be remembered, however, that the energy loss depends on the pulse length at the cavity, as does the interpretation of the data. Any distortion of the signal as it proceeds from the cavity to the output detector will, therefore, introduce errors. Al though these can, in principle, be corrected for if one knows sufficiently well the pulse response of the system from the cavity to the output, it would be good to keep such corrections sma11 -- by which I mean that the resolution time (rise-time) of the system should be quite a bit less than $\tau$, and, preferably, about equal to the resolution time of the detecting oscillograph. 


\section{Oscillograph}

As we shall see below, the interpretation of the measurements depends in some detail on the shape of the signals at the output of the bench set-up. We would like, ideally, the oscillograph to give a faithful record of the time function of the original pulse and of the modified pulse as they leave the cavity structure. It is not possible to make in advance any precise statement on the magnitude of the error that will be introduced by the resolution time of the oscillograph. We would need to know something about the signals that will be obtained from any particular cavity structure. I estimate, however, that, if the resolution time (full-width) of the oscillograph is about one-fourth of the pulse length $\tau$, the uncorrected error in the energy loss measurement wi11 be about 10\%. Oscillographs are available with a resolution time of about 30 to 40 picoseconds, which should be more than adequate.

\section{Interpretation of the Data}

The raw data of the measurements will be two time functions recorded by the oscillograph. One is the "original" current pulse $i_{0}(t)$ that passes through the system in the absence of the cavity, and the other is the "modified" current pulse $i_{m}(t)$ that is observed when the cavity is in place. I sha11, for the moment, consider all currents and voltages as they appear at some arbitrary point in the transmission line of the beam chamber after the cavity but before entering the tapered section. See Fig. 3. The magnitudes wi11, of course, be different after transformation to a different impedence level by the tapered matching section. Let me now define the 
"secondary" current $i_{a}(t)$ as the difference between the original pulse and the modified one:

$$
i_{a}(t)=i_{0}(t)-i_{m}(t)
$$

This current is the one generated by the "secondary" fields that result from the original pulse.

Let's let $U$ stand for the total energy that would be lost by a relativistic particle bunch in traversing the cavity. For any cavity, $U$ is proportional to the square of the charge $q$ in the bunch:

$$
U=k q^{2},
$$

and it is the constant $k$ that we wish to determine by the bench test. The average energy lost per particle, $u_{0}$, is related to $k$

$$
u_{0}=k q e .
$$

It is shown in Appendix $B$ that $k$ can be obtained from $i_{0}(t)$ and $i_{a}(t)$ by the equation

$$
k=\frac{2 R_{0}}{q_{0}^{2}} \int i_{a}(t) \cdot i_{0}(t) d t+\delta k,
$$

where $R_{0}$ is the characteristic resistance of the transmission line, and $q_{0}$ is the total charge in the pulse as given in Eq. (2). The correction term sk acknowledges the imperfection of our model of the energy loss for a bunch. This correction will be strictly zero only in the limit that 
$i_{d}(t) \rightarrow 0$ for any $t$. I estimate that the relative correction $\delta k / k$ will be roughily given by

$$
\frac{\delta k}{k} \approx \frac{I_{a}}{I_{0}},
$$

where $I_{a}$ is a "typical" value of $i_{a}(t)$-- say, the value at the center of $i_{0}(t)$-- where $i_{0}(t)$ has the value $I_{0}$.

There is an alternative expression, which is, in theory, equivalent to Eq. (10), although it involves an additional instrumental complication. Suppose that, besides measuring $i_{0}(t)$ and $i_{a}(t)$, we were to measure also $i_{b}(t)$, the signal "reflected" from the cavity back toward the pulse source. Then the energy loss constant $k$ can also be obtained from

$$
k=\frac{2 R_{0}}{q_{0}^{2}} \int\left[i_{a}^{2}(t)+i_{b}^{2}(t)\right] d t+\delta k .
$$

I expect this expression to be less usefur, in practice, than Eq. (10), because the integral must be extended over the rather long decay times of $i_{a}(t)$ and $i_{b}(t)$ where these currents will be small in magnitude and probably only imprecisely known. Eqs. (10) and (12) are connected by the conservation of energy. See Appendix B.

If Eq. (10) is used to determine $k$, the error in the value obtained will depend in part on the precision with which the integral can be evaluated, and, in part, on the "error" $\delta k$. The error in the integral will be determined primarily by the error in $i_{a}(t)$-- which is, you will remember -the difference between $i_{0}(t)$ and $i_{m}(t)$. This error will be less; the larger is $i_{a}(t)$. On the other hand, the error $\delta k$ is proportional to 
the magnitude of $i_{a}(t)$. Although $\delta k$ is a systematic error -- and $a$ correction of it can, in principle, be estimated -- let's assume, for the moment, that it has an unpredictable uncertainty about equal to itself. In this circumstance, it is clear that the measurement will give its maximum accuracy for a particular optimum magnitude of $i_{a}(t)$-- which is controllable by the choice of the size of the central wire. From an elementary and approximate error analysis, I estimate that the highest accuracy will be obtained for $k$, when $\delta k / k \approx \Delta k / k$, which means that

$$
I_{\mathrm{a}} \approx \sqrt{\mathrm{I}_{0} \Delta \mathrm{I}_{\mathrm{a}}}
$$

where $I_{a}$ is the typical value of $i_{a}(t)$ defined earlier, and $\Delta I_{a}$ is the absolute error in the determination of $i_{a}(t)$. When Eq. (13) holds, the error $\Delta k$ (total) has its minimum value

$$
\frac{\Delta k(\text { tota } 1)}{k} \approx \sqrt{\frac{2 \Delta I_{a}}{I_{0}}} .
$$

If $\Delta \mathrm{I}_{\mathrm{a}} / \mathrm{I}_{0}$ is about one percent, then the relative error in $k$ would, under optimum conditions, be about $14 \%$.

Unfortunately, the constant $k$ does not necessarily give the energy loss of most significance to the operation of a storage ring. It was pointed out in Ref. 1 that it is not the total energy loss $u_{0}$ that is of primary concern but rather the loss $S$ to the parasitic modes -- which is that part of $u_{0}$ that remains when the energy loss $u_{00}$ to the fundamental mode is subtracted out. Making the same separation here, we can write 


$$
k=k_{0}+k_{s} \text {, }
$$

where $k_{0} q^{2}=u_{00}$ and $k_{s} q^{2}=S$. Fortunately, $k_{0}$ can be determined directly from bench measurements of the standard $r f$ type to reasonable accuracy -perhaps to $5 \%$ or better. It is also determined by LALA, the computer program used for designing the cavities. The magnitude of $k_{0}$ is rather large, perhaps $30 \%$ to $50 \%$, or more, of the total $k$. If $k_{s}$ and $k_{0}$ are comparable, the error in the determination of $k_{s}$ will be doubled -- to perhaps $30 \%$ or $40 \%$. We must remember, however, that the parasitic energy loss to the rf accelerating cavities is only of interest to us if it is not small -- and that means in comparison with $u_{00}$. If $k_{s}$ is at least somewhat smaller than $k_{0}$, the error in $k_{s}$ becomes

$$
k_{S} \approx \sqrt{\frac{2 \Delta I_{a}}{I_{0}}} k_{0}
$$

We can expect to know the absolute magnitude of the energy loss $S$ to an accuracy about $15 \%$ or so of the fundamental loss $u_{0}$. For PEP, $u_{0}$ is about $5 \mathrm{MeV}$ (for the whole ring) so we can hope to know $S$ to an accuracy of about $0.75 \mathrm{MeV}$, which is sufficiently small in comparison with the energy lost each turn to synchrotron radiation (26 MeV at $15 \mathrm{GeV}$ ) that it becomes insignificant. We may conclude that the proposed method will provide sufficient accuracy for the design of the ring.

The comments of the preceding paragraph apply only to the rf accelerating cavities. There is also some concern about the hundreds of "incidental" cavities in the ring -- namely, the various transition chambers in the vacuum system. For these cavities, the total $k$ gives the relevant loss, 
and the predicted accuracy should be sufficient to show the general magnitude of the loss -- and, if it should turn out to be serious, serve to permit the control of design changes to reduce the losses to an acceptable amount.

One further comment about Eq. (10). In all of the discussion above, it was assumed that the currents were those flowing in coaxial transmission line formed by the wire and the "normal" vacuum chamber. This line is matched to the detector by a tapered matching section which should -- if properly designed -- act as an ideal transformer with a current-transformation ratio $M$. The product of the two currents will be larger by the factor $M^{2}$ and the proper matching resistance will be smaller by the same factor. Equation (10), therefore, can also be used with all quantities referred to the input of the detector.

\section{Expected Signal}

It was estimated ${ }^{1}$ that a beam bunch containing $1.5 \times 10^{12}$ particles might lose an energy of $15 \mathrm{MeV}$ per particle in traversing the 120 cavities of the PEP electron-positron ring. Such a loss corresponds to an energyloss constant $k$ of $5.2 \times 10^{11}$ volt/coul for one PEP cavity. This estimate has, however, a large uncertainty. My personal guess is that it might be half as large or less. It cannot be any smaller than the known loss $k_{0}$ to the fundamental mode, so the answer will probably lie between the two values:

$$
\begin{array}{ll}
\mathrm{k}(\text { est }) & =5.2 \times 10^{11} \mathrm{volt} / \mathrm{cou} 1 \\
k_{0} & =1.6 \times 10^{11} \mathrm{volt} / \mathrm{cou} 1 .
\end{array}
$$


I now show that the proposed set-up will give reasonable signals for such energy losses.

First, let me say what I expect for the time-variation of the secondary current pulse $i_{a}(t)$. For a not-too-pathological cavity, I expect that the general shape of the signals $i_{m}(t)$ and $i_{a}(t)$ in the neighborhood of the original pulse $i_{0}(t)$ might be as shown in Fig. 4. I am, of course, only guessing about what the shape of $i_{a}(t)$ might be -- particularly after its initial rise. Anyway, let's assume that they might be as shown.

The integral of Eq. (10) can be estimated in the following way. The original current $i_{0}(t)$ can be approximated by a rectangular pulse of magnitude $I_{0}$ and duration $\tau$. The secondary current $i_{a}(t)$ can be replaced by its typical value $I_{a}$ during the interval $\tau$. With this approximation, the integral is roughly equal to $I_{0} I_{a} \tau$, and the rest of Eq. (10) then tells us that

$$
\frac{I_{a}}{I_{0}} \approx \frac{\tau k}{2 R_{0}}
$$

The constants $\tau$ and $k$ are determined for us (though $k$ is unknown), so the only free parameter is $R_{0}$, the characteristic resistance of the coaxial transmission line. This resistance is determined primarily by the size of the central wire placed in the beam tube. Let's approximate the beam tube by a cylinder of inner radius $\underline{a}$; if the wire has a radius $r$,

$$
R_{0}=\frac{1}{2 \pi} \sqrt{\frac{\mu_{0}}{\varepsilon_{0}}} \ln \frac{a}{r}=\text { (60 0hms) } \ln \frac{a}{r} \text {. }
$$

Equations (18) and (19) justify the statement made in Section 2 that $I_{a}$ 

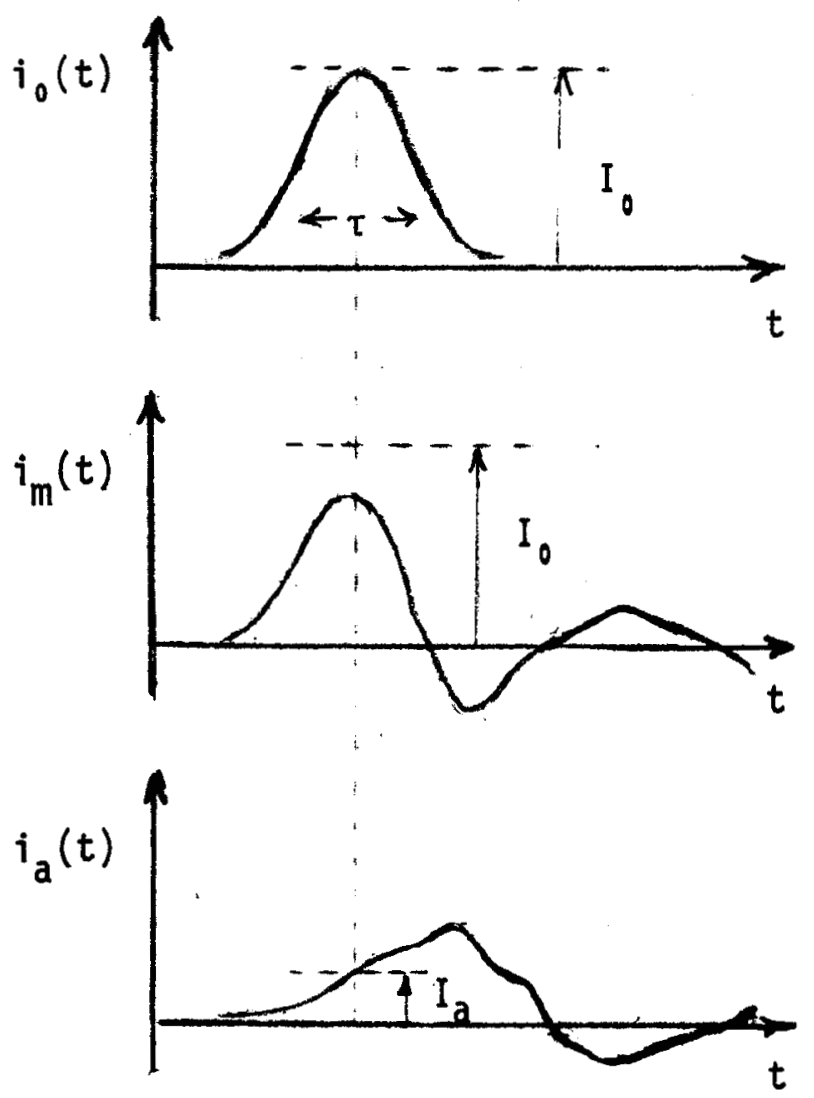

Fig. 4. Form of Expected Signals 
can be made small in comparison with $I_{0}$.

First, let's look at what might be a "convenient" value for $R_{0}$. Suppose that for mechanical reasons we were to choose a wire whose radius was 1 millimeter, and that we take for a the inner radius of the PEP cavities, $6 \mathrm{~cm}$. Then $\mathrm{R}_{0}=246$ ohms. Taking, further, that $\tau=3.3 \times 10^{-10} \mathrm{sec}$ and $k=5.2 \times 10^{11} \mathrm{volt} / \mathrm{coul}$, so that $k \tau=172 \mathrm{ohms}$,

$$
\frac{I_{a}}{I_{0}} \approx 0.34
$$

The expected secondary signal is about one-third the size of the original pulse, and is a little large for the optimum measurement. This value of $R_{0}$ would, however, be about right if the actual value of $k$ turns out to be about one-haif of the estimated one.

For an optimum measurement, the size of the center wire should be chosen so that the expected signal satisfies Eq. (13). Then

$$
\left(R_{0}\right)_{\text {opt }}=\frac{k \tau}{2} \sqrt{\frac{I_{0}}{\Delta I_{a}}} .
$$

Or, using Eq. (19),

$$
\left(\frac{r}{a}\right)_{\text {opt }}=\exp \left\{-\frac{k \tau}{1200 \mathrm{hms}} \sqrt{\frac{I_{0}}{\Delta I_{a}}}\right\} \text {. }
$$

Taking $\mathrm{kT}=172$ Ohms, as before, and $I_{0} / \Delta I_{a}=100$, we would have

$$
\left(\frac{r}{a}\right)_{\text {opt }}=6.0 \times 10^{-7} .
$$


The optimum wire radius is $10^{-7}$ centimeters -- which is, I am afraid, not very practical. It is clear that the optimum measurement cannot be realized if the loss factor $k$ is too large. If $k$ for a PEP cavity is near its minimum value $k_{0}$, for which $k_{0} \tau=52$ ohms,

$$
\left(\frac{r}{a}\right)_{\text {opt }}=0.012 \text {, }
$$

or $r \approx 0.8$ millimeters -- a quite reasonable value.

It appears that we have been quite lucky in that a reasonable wire size is about what would give an optimum measurement. (Although the "luck" is from the same roulette wheel that makes the expected energy loss neither disastrous nor insignificant!) I will show in the next Section that other considerations also limit the wire size to something reasonable.

\section{Limitations on the Choice of the Center Conductor}

In the preceding Section, it was shown that the choice of the size of the center conductor will influence the accuracy of the measurement of the cavity loss, and that, in principle, the wire size can be chosen to obtain a minimum error. The analysis of Section 6, and, in particular, the reasoning behind Eq. (13), is inadequate in two respects. First, the assumption has been made -- as mentioned in Section 2 -- that the basic energy loss mechanism is not drastically changed by the presence of the center conductor. Second, pulse distortions due to the resistance of the center wire have, so far, been neglected. I will now examine in a little more detail these assumptions. 
In Appendix A, an approximate equivalent circuit is derived for a cavity with an added central wire, and the circuit is used to estimate the change in the natural frequency of the fundamental mode and to determine the effective quality factor $Q$ for that mode. It is shown that for any given cavity, that is not too strange, the frequency shift with the wire present can be written as

$$
\frac{\Delta \omega}{\omega_{0}} \approx \frac{K_{1}}{\ln a / r} \text {, }
$$

where $k_{1}$ is an electrical property of the cavity, a is the radius of the beam pipe and $r$ the radius of the wire. The frequency shift decreases with decreasing $r$, but very slowly. For the PEP cavities, $K_{1}=0.50$ and $a=6.0 \mathrm{~cm}$. Taking $r=0.1 \mathrm{~cm}$, we get $\Delta \omega / \omega_{0} \approx 0.1$ as mentioned earlier. The frequency shift is smal1, and will not get much larger for any reasonable $r \ll a$.

The same analysis gives an expression for the quality factor that is

$$
Q \approx K_{2} \ln \frac{a}{r} \text {, }
$$

where $K_{2}$ is again a number associated with the free cavity. For the PEP cavities, $K_{2} \approx 2.0$. And for the same $r$ and a used above, $Q=8.2$. Again, we see that $Q$ changes slowly with $r$. Eqs. (25) and (26) both confirm the intuitive guess that the cavity will be the least disturbed by the smallest wire.

There is, however, one important effect that gets worse for smaller 
wires. It is the distortion of the pulse that arises from resistive losses in the wire. The resistance of the wire causes an attenuation of the pulse as it travels along the wire, and, since the resistivity is frequencydependent, there will be different attenuations for the different frequency components in the pulse, leading to a distortion of the pulse. A small amount of distortion will not be serious because the "original" pulse shape is measured including these distortions. Large distortions will, however, introduce errors because of the change in pulse shape from the cavity to the detector.

A Fourier component whose amplitude is $A(\omega)$ will be attenuated with distance $x$ along the wire according to $\mathrm{e}^{-\alpha(\omega) x}$. So long as $r \ll a$, the attenuation coefficient $\alpha(\omega)$ is given by

$$
\alpha(\omega)=\frac{R_{S}(\omega)}{4 \pi R_{0} r} \text {, }
$$

where $R_{S}$ is the surface resistivity of the wire at the frequency $w$, and $R_{0}$ is the characteristic resistance of the transmission line. The surface resistivity depends on frequency as

$$
R_{S}(\omega)=n \sqrt{\omega}
$$

where, for example, $n=1.04 \times 10^{-7} 0 \mathrm{hm} / \mathrm{sec}^{\frac{1}{2}}$ for copper. ${ }^{2}$ The characteristic resistance is given by Eq. (19).

Let's evaluate $\alpha(\omega)$ at the typical frequency $\omega_{0}$ that was defined in Section 4. Assuming a copper wire, 


$$
\alpha\left(\omega_{0}\right)=\frac{K_{3}\left(\omega_{0}\right)}{r \ln a / r},
$$

with $k_{3}\left(\omega_{0}\right)=1.08 \times 10^{-5}$ for a $10-\mathrm{cm}-$ long pulse. Taking $a=6.0 \mathrm{~cm}$ and $r=0.1 \mathrm{~cm}$, as before,

$$
\alpha\left(\omega_{0}\right)=2.5 \times 10^{-3} \text { meter }^{-1} .
$$

The attenuation is only one-quarter of a percent in one meter -- clearly quite negligible. Notice, however, the inverse dependence of $\alpha$ on $r$. Suppose that the length of the bench set-up is a meter or two long, and let's say that we would not like an $\alpha\left(\omega_{0}\right)$ greater than about 0.1 . The minimum wire size would then be about $1.2 \times 10^{-3} \mathrm{~cm}$. Such a small wire might be difficult to handle, so the resistive loss is not likely to be a serious 1 imitation.

It would appear that wire sizes somewhere between perhaps $10^{-2} \mathrm{~cm}$ and $1 \mathrm{~cm}$ might be used -- the choice being made to optimize the accuracy of the measurements as described in Section 6 .

\section{Synthetic Pulse Method}

A11 of the preceding discussion was based on the assumption that the current pulse launched along the central wire would have a time-shape that simulates the current of a circulating bunch. I would like now to point out that this requirement can be dropped provided that the whole system is certain to be completely linear at all of the relevant frequencies and amplitudes -- that is, if the oscillograph is linear, and if all of the metalic contacts have linear resistances. With a strictly linear 
system, the experimental measurements can be made with almost any reasonable pulse, and then analysed to obtain the coefficient $k$ appropriate to an idealized Gaussian pulse of any length. By a "reasonable" pulse, I mean, of course, a pulse that has significant Fourier components at the relevant frequencies -- specifically, those in the neighborhood of $w_{0}$ for the desired bunch length.

Suppose we have a pulser that gives a time-dependent current $i_{0}^{*}(t)$ which is some sort of a short impulse of length $\tau^{*}$ that might be, say, quite a bit less than the bunch length $\tau$ which we wish to simulate. We can, as has been assumed until now, pass this pulse through a filter to generate an (approximately) Gaussian pulse $i_{0}(t)$ of width $\tau$, which is then used for the measurements. When we do this, the pulse $i_{0}(t)$ is transformed into the pulse $i_{m}(t)$ by the response of the cavity system. Now Tet's look at what is happening in the following way. The modified pulse $i_{m}(t)$ is the "output" obtained when the "input" $i_{0}^{*}(t)$ is applied to the cascaded system to two "filters" - - the pulse-shaping filter and the cavity system. But, as is well known, with linear systems the sequence of the two filters doesn't matter (exchanging the order of two linear operators leaves the result unchanged). So we could just as well place the pulse-shaping filter after the cavity system, rather than before.

What I want to suggest is that we go one step farther and omit the pulse-shaping filter entirely. Instead, we can perform the equivalent operation mathematically on the signals after they have been recorded by the oscillograph.

Let me define the linear operators $O_{p}$ and $O_{C}$, that are to represent 
the pulse-shaping network and the cavity system so that

$$
\begin{aligned}
& i_{0}(t)=0_{P} \cdot i_{0}^{*}(t) \\
& i_{m}(t)=0_{C} \cdot i_{0}(t) \\
& i_{m}(t)=0_{C} \cdot\left\{0_{P} \cdot i_{0}^{*}(t)\right\} .
\end{aligned}
$$

Since the operators are linear, I can reverse their order; then $i_{m}(t)$ is a)so given by

$$
i_{m}(t)=0_{p} \cdot\left\{0_{C} \cdot i_{0}^{*}(t)\right\} .
$$

Let me now define $i_{m}^{*}(t)$ as the signal that would be recorded by the oscillograph if the pulse $i_{0}^{\star}(t)$ were applied directly to the cavity system. Then

$$
i_{m}^{*}(t)={ }_{C} \cdot i_{0}^{*}(t)
$$

and

$$
i_{m}(t)=0_{p} \cdot i_{m}^{*}(t)
$$

And, in fact, the operation $0_{p}$ can equally well be performed on the recorded signal after it is recorded.

Now recall that we are not primarily interested in the function $i_{m}(t)$, but in the difference function $i_{a}(t)$, where

$$
i_{a}(t)=i_{0}(t)-i_{m}(t) .
$$

Using Eqs. (31) and (36), we can rewrite the difference as

$$
i_{a}(t)=o_{p} \cdot\left\{i_{0}^{*}(t)-i_{m}^{*}(t)\right\} \cdot
$$


Now let's define $i_{a}^{*}(t)$

$$
i_{a}^{*}(t)=i_{0}^{*}(t)-i_{m}^{*}(t)
$$

This signal is just the difference of the two signals (the original signal and the modified signal) that would be recorded if the pulse $i_{0}^{*}(t)$ were used for the input signal. So if we use as our basic data the two functions $i_{0}^{*}(t)$ and $i_{a}^{*}(t)$, we obtain $i_{0}(t)$ and $i_{a}(t)$ from Eq. (31) and the corresponding equation for $i_{a}(t)$, which, from Eq. (38), is

$$
i_{a}(t)=0_{p} \cdot i_{a}^{*}(t)
$$

The operation $0_{p} \cdot i(t)$ can be written as

$$
O_{p} \cdot i(t)=\int_{-\infty}^{t} G\left(t-t^{\prime}\right) \cdot i\left(t^{\prime}\right) d t^{\prime},
$$

where $G(t)$ is the response of the pulse-shaping filter to the ideal impulse $\delta(t)$. Once we have $i_{0}^{*}(t)$ and $i_{a}^{*}(t)$, the operation $o_{p}$ is merely a numerical integration.

What would be the advantage of using the synthetic-pulse method? For one, it might make the experiment easier if one did not have to shape the pulse from the pulser. More importantly, it would allow obtaining the energy loss as a function of bunch length by a numerical computation, rather than by running experiments for each bunch length. Most importantly, however -it might permit an increase in the precision of the measurements. I have not made an analysis of the errors that would be expected with the synthetic pulse method, but I believe that the accuracy could be improved notably provided that (a) the system is indeed linear, and (b) the pulse length $\tau^{*}$ 
of $i_{0}^{*}(t)$ is somewhat shorter than $\tau$.

This improvement would come about for the following reason. The requirement that $i_{a} / i_{0}$ be significantly less than 1 in the middle of $i_{0}(t)$ would still apply to this ratio, and not to the ratio $i_{a}^{*} / i_{0}^{*}$. So long as the basic limitation on the accuracy of the measurement rests on the accuracy of the determination of $i_{a}$-- which would, I assume, be the same for $i_{a}^{*}$-an increase in the (relative) magnitude of $i_{a}^{*}$ would result in an improved determination of the energy loss constant $k$.

\section{Conclusions}

A bench measurement has been proposed that would permit a measurement of the energy loss of a stored particle beam to a cavity in the vacuum chamber. An estimate of the accuracy of the method indicates that the energy loss to the parasitic modes of the rf accelerating cavity can be determined with an error that will be $15 \%$ or less of the loss to the fundamental mode. For other incidental cavities in the ring (vacuum boxes and the 1 ike), the losses could be measured to a relative error of $15 \%$ or better. These accuracies are probably more than are needed for the design of PEP.

The bench set-up would permit relatively easy measurements of the loss to all of the structures that will be used in the vacuum chambers. And will, if necessary, provide a convenient way of experimenting with design modifications that would reduce the loss.

It would, of course, still be useful to make measurements of the cavity loss in SPEAR-2; the results of which could be compared with the results of a bench measurement to confirm -- or question -- the quality of the bench measurement. 


\section{Acknowledgement}

I am grateful for illuminating conversations with Matt Al len, Dick Helm, Ron Koontz, Greg Loew, Roger Miller, Andy Sabersky and Perry Wilson. The measurement referred to in Section 2 was carried out by Matt Allen. Cathy Nissen made essential contributions to the preparation of this report.

\section{References}

1. M. Sands, "Energy Loss to Parasitic Modes of the Accelerating Cavities", PEP-90 (Ju1y 1974).

2. See, for example, Ramo and Whinnery: Fields and Waves in Modern Radio, Weley (1944). 
APPENDIX A.

Equivalent Circuit of a Cavity with a Central Wire

Each mode of a resonant cavity is characterized by three parameters. I find it convenient to use the set $\omega_{0}$, the natural frequency, $r_{0}$, the intrinsic impedance, and $Q$, the loss coefficient. (The so-called "shunt resistance" $R_{S}$ of a cavity is $\left.2 r_{0} Q_{.}\right)$For a single PEP cavity, the values are, for the fundamental mode:

$$
\begin{aligned}
& \omega_{0}=2.25 \times 10^{9} \mathrm{sec}^{-1} \\
& r_{0}=1400 \mathrm{hms} \\
& Q_{0}=28,000 .
\end{aligned}
$$

It is convenient for some purposes to represent the cavity, for frequencies near resonance, by an equivalent RLC circuit. If we take the circuit to be a parallel combination of all three, as shown in Fig. A-1(a), then

$$
\begin{aligned}
L=r_{0} / \omega_{0} & =6.2 \times 10^{-8} \text { henry } \\
C=1 / r_{0} \omega_{0} & =3.2 \times 10^{-12} \text { farad } \\
R_{\|}=r_{0} Q_{0} & =4.0 \times 10^{6} \text { Ohms }
\end{aligned}
$$

When the wire is inserted along the axis of the cavity, we can represent its effect by adding a few lumped impedances as shown in Fig. A-1(b). The inductance $L$ ' represents the self-inductance of the wire, $C$ ' represents the "stray" capacitance of the exposed wire to the edges of the cavity and $R_{0}$ is the characteristic resistance of the coaxial transmission line formed by the wire with the vacuum pipe on either side of the cavity. The composite sketch of Fig. A-1(c) illustrates the relationship of these elements 

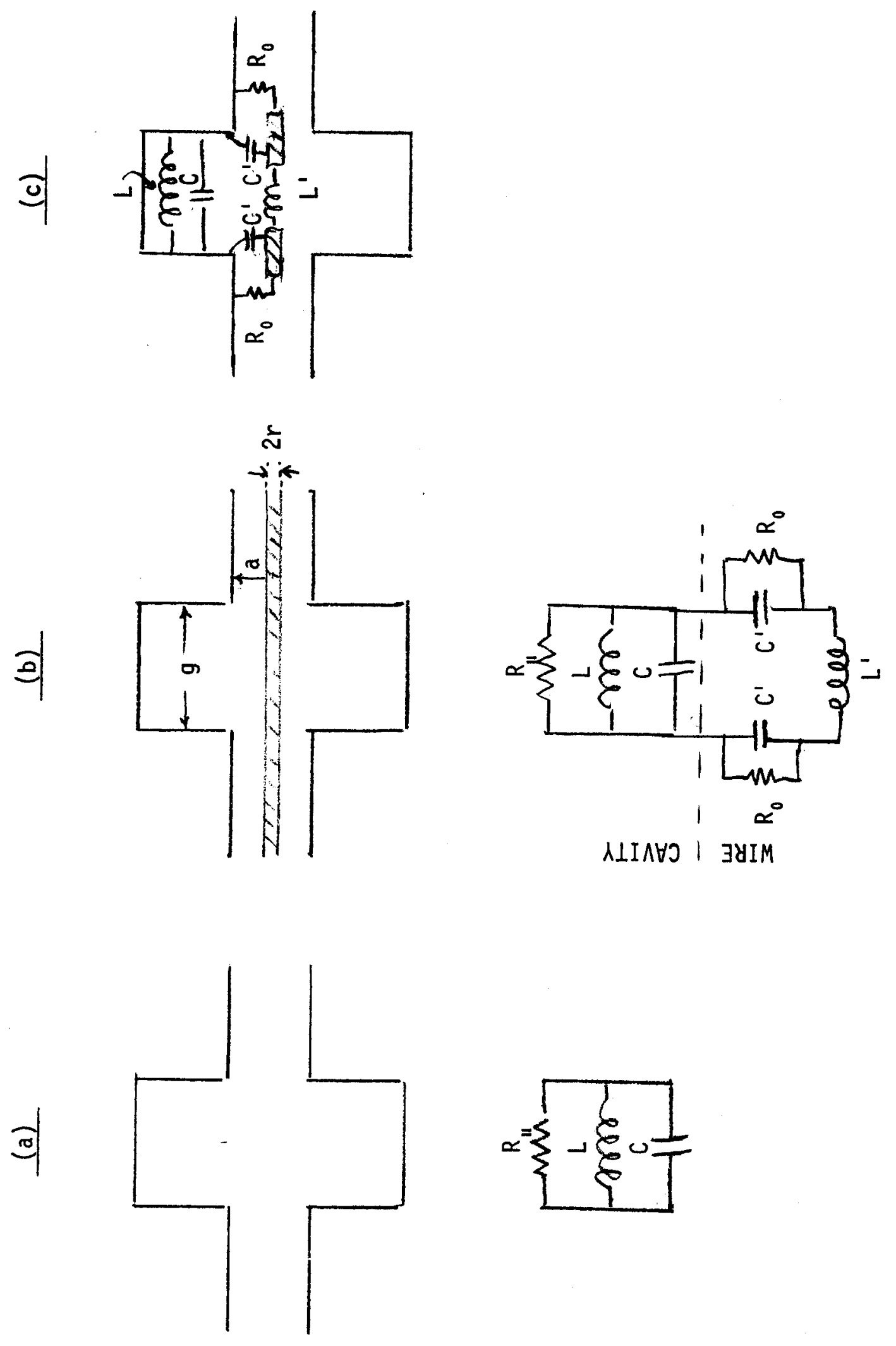

Fig. A-1. Equivalent Circuit of Cavity with Wire 
to the geometry of the system.

The values to be taken for these impedances depend on the following dimensions, which are also shown in Fig. $A-1(b)$ :

$$
\begin{aligned}
& r \text {; wire radius } \\
& \text { a; pipe radius } \\
& g \text {; cavity length. }
\end{aligned}
$$

The resistance $R_{0}$ is given by

$$
R_{0}=\frac{1}{2 \pi} \sqrt{\frac{\mu_{0}}{\varepsilon_{0}}} \text { ln } \frac{a}{r}=60 \text { ln } \frac{a}{r} \text { Ohms. }
$$

I estimate that the inductance $L^{\prime}$ is approximately equal to the inductance of a length $g$ of the central wire. Then

$$
L^{\prime}=\frac{\mu_{0}}{2 \pi} g \ln \frac{a}{r} \text {. }
$$

I estimate $C^{\prime}$ to be approximately equal to the capacitance of a coaxial capacitor inner radius $r$, outer radius $a$, and also of length $a$. Then

$$
C^{\prime}=\frac{2 \pi \varepsilon_{0} a}{\ln a / r} \text {. }
$$

To 17 lustrate the general magnitudes of these impedances, let's take the example of a PEP cavity with a central wire whose radius is 1 millimeter. For this example we get the following numbers: 


$$
\begin{aligned}
& r=10^{-3} \quad \mathrm{~m} \\
& a=0.06 \quad \mathrm{~m} \\
& g=0.25 \quad \text { ohms } \\
& R_{0}=246 \quad \text { henry }\left(X_{L}^{\prime}=4610 \mathrm{hms}\right) \\
& \left.L^{\prime}=2.05 \times 10^{-7}\right) \\
& C^{\prime}=8.1 \times 10^{-13} \text { farad }\left(X_{C}^{\prime}=5490 \mathrm{hms}\right)
\end{aligned}
$$

I have also shown the impedances $X_{L}^{\prime}=\omega L^{\prime}$ and $X_{C}^{\prime}=1 / \omega C^{\prime}$ at the cavity's unperturbed resonance frequency $\omega_{0}$.

Notice that for the illustrative example chosen, $X_{C}^{\prime}$ is about twice as big as $R_{0}$. The ratio $X_{C}^{1} / R$ is equal to $\lambda_{0} / a$, where $\lambda_{0}$ is the reduced wavelength of the resonant frequency $\omega_{0}$, and is independent of the wire size $r$. I shall assume that this ratio is large enough that I can ignore $C^{\prime}$. Taking $C^{\prime}$ into account would be a bit tedious, and, I would guess, perhaps of doubtful use. I sha11, therefore, estimate the perturbation of the equivalent resonant circuit of the cavity by taking into account only the effect of shunting the cavity by the series combination of the inductance $L^{\prime}$ and the resistance $2 R_{0}$.

The change in the resonant frequency is approximately the change that would occur by replacing $L$ of the cavity by the parallel combination of $L$ and $L^{\prime}$. For $L^{\prime} \gg L^{\prime}$

$$
\frac{\Delta \omega}{\omega_{0}} \approx \frac{1}{2} \frac{L}{L^{\prime}}=\frac{1}{2} \frac{r_{0}}{X_{L}^{\prime}} .
$$

Using Eq. (A-4), 


$$
\frac{\Delta \omega}{\omega_{0}} \approx \frac{\pi r_{0}}{\omega_{0} \mu_{0} g} \cdot \frac{1}{\ln a / r} .
$$

For the PEP cavities, the first term is 0.50 .

The energy dissipation in the cavity resistance $R_{\|}$is negligible, so the $Q$ of the combined circuit will be given by the loss in the resistance $2 R_{0}$. I get that

$$
Q \approx \frac{2 R_{0}}{r_{0}}\left(1+\alpha^{2}\right) \text {, }
$$

where

$$
\alpha=\frac{X_{L}^{\prime}}{2 R_{0}} \text {. }
$$

Using Eqs. $(A-3)$ and $(A-4)$,

$$
\begin{gathered}
Q \approx \frac{120 \text { Ohms }}{r_{0}}\left(1+\alpha^{2}\right) \ln \frac{a}{r} \\
\alpha=\frac{\omega_{0} g}{2 c} .
\end{gathered}
$$

For the PEP cavities, $\alpha=1.16$, and

$$
Q \approx 2.0 \ln \frac{a}{r} \text {. }
$$


APPENDIX B.

\section{Energy Loss Formulae}

A current pulse $i(t-x / c)$ travelling along a coaxial transmission line with characteristic resistance $R_{0}$ has a potential difference $v$ equal to $R_{0} i$, and a linear charge density $\lambda=i / c$, where $c$ is the propagation velocity. The energy contained in the pulse is

$$
U=\int i v d t=R_{0} \int i^{2} d t
$$

evaluated at any $x$. In the following, I shall take $x=0$ at the evaluation point.

In the experimental set-up, the energy in the system before the pulse arrives at the cavity -- when the current is $i_{0}(t)--$ is

$$
U_{1}=R_{0} \int i_{0}^{2}(t) d t \text {. }
$$

Later, there is the modified signal $i_{m}(t)=i_{0}(t)-i_{a}(t)$ travelling to the right and the reflected pulse $i_{b}(t)$ going to the left. See Section 6 . The total energy is

$$
U_{2}=R_{0} \int\left[i_{0}(t)-i_{a}(t)\right]^{2} d t+R_{0} \int i_{b}^{2}(t) d t .
$$

From the conservation of energy (and neglecting any energy dissipation other than in the terminations of the coaxial lines), $U_{2}=U_{1}$, so

$$
-2 R_{0} \int i_{0}(t) i_{a}(t) d t+R_{0} \int\left[i_{a}^{2}(t)+i_{b}^{2}(t)\right] d t=0 .
$$

I would like to identify the magnitude of the first term of this equation 
as the energy "lost" from the initial pulse. The second term, which is equal to the first, I would like to identify as the energy "deposited" in the cavity, and subsequently released to the coaxial lines.

I shall now show that the first term corresponds to the energy given up to the cavity by a relativistic particle bunch with the same current dependence. Consider an element of charge $\Delta q$ in the bunch as it passes through the cavity. See Fig. A-2. The energy gained by the charge $\Delta q$ as it traverses the distance $\mathrm{dx}$ is $\Delta \mathrm{q} \boldsymbol{E}_{\mathrm{q}} \mathrm{dx}$, where $\boldsymbol{E}_{\mathrm{q}}$ is the electric field strength at the charge. The total energy received by $\Delta q$ when it has moved well beyond the cavity is

$$
\Delta U=\Delta q \int \varepsilon_{q} d x
$$

Now consider what happens when a current pulse on the wire passes through the cavity. If the current pulse does, to first order, not change as it goes through the cavity, an element of charge $\Delta \mathrm{q}$ will experience the same electric field $\mathcal{E}_{q}$ and will gain the energy given in Eq. (B-5). For a current pulse, however, the energy in an element of charge $\Delta q$ is related to the current at $\Delta q$ and so the current must change. The rate of energy flow past any point is $R_{0} i^{2}$, so if the charge $\Delta q$ arrives at any $x$ in the time $\Delta t$, the energy carried by $\Delta q$ is $R_{0} i^{2} \Delta t$. For a small change in the current, the change in energy is

$$
\Delta U=2 R_{0} i \Delta i \Delta t=\Delta q \cdot 2 R_{0} \Delta i
$$

since $i \Delta t$ is just $\Delta q$. This expression must be the same as Eq. $(B-5)$, from which we get that 


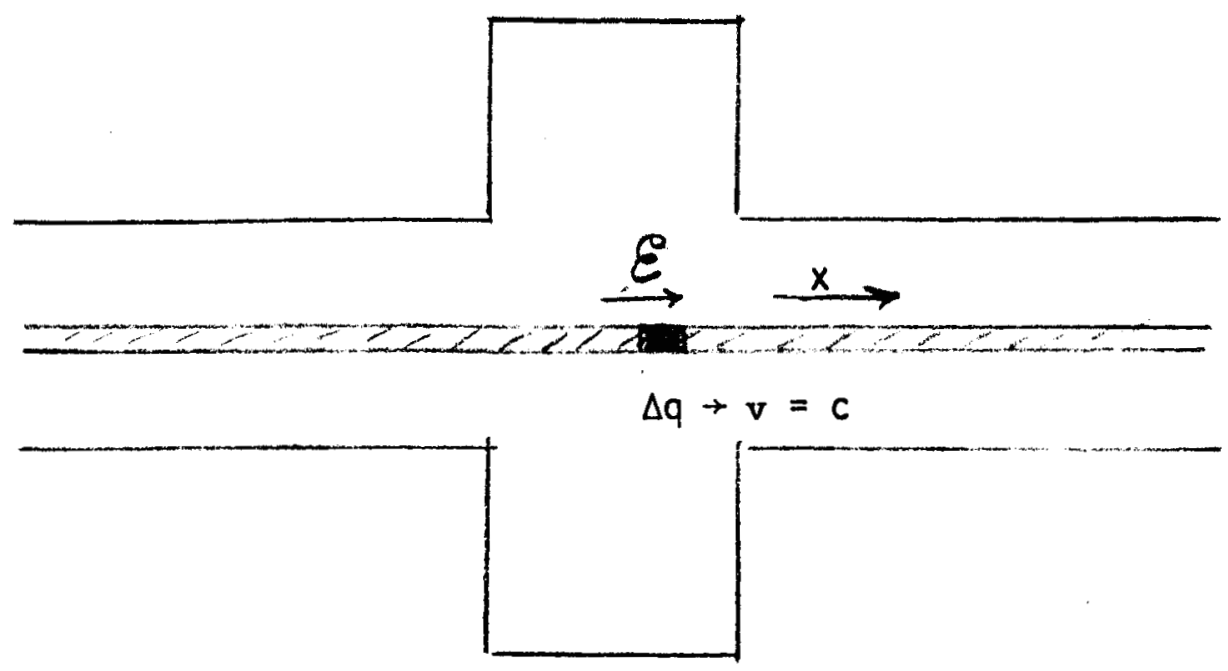

Fig. A-2. Secondary Electric Field $\varepsilon$ Acting on

a Moving Charge Element $\Delta \mathrm{q}$ 


$$
\int \varepsilon_{q} d x=2 R_{0} \Delta i_{q}
$$

That is, the energy given to the charge element $\Delta q$ is proportional to the change $\Delta i$ at $\Delta q$. Of course, energy is actually removed from the pulse, so $\Delta i$ is negative. The total energy lost by the bunch is obtained from the sum of terms like Eq. (B-5) for each $\Delta q$ in the bunch. Writing $\Delta q=i \Delta t$ the energy lost is

$$
U_{\text {loss }}=-2 R_{0} \int i \cdot \Delta i d t
$$

Recalling that $i_{a}(t)$ was defined to be the change in $i_{0}(t)$ due to its passage through the cavity, this equation is the same as the first term in Eq. (B-4). So to the approximation that $\Delta i \ll i$, Eq. (B-8) represents the energy lost by a bunch whose time dependence is $i(t)$.

It may be worth pointing out that Eq. (B-8) can also be derived by writing directly the currents induced in the wire by the secondary electric field $\varepsilon$. Consider what happens if an externally impressed electric field

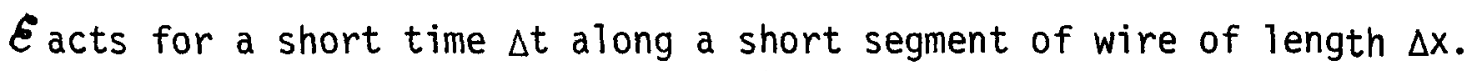
From the "circuit" point-of-view, there will be a "potential difference" (or e.m.f.) in the wire equal to $\boldsymbol{E} \Delta x$. Since the transmission like looks like a resistance $R_{0}$ in each direction, a current pulse $\Delta i_{a}=\varepsilon \Delta x / 2 R_{0}$ will be generated which travels in one direction and an equal pulse of opposite polarity $\Delta i_{b}$ that travels in the opposite direction. Focussing on the pulse $\Delta i_{a}$, it will have a duration $\Delta t$, and will travel along the wire with the speed c. If electric fields are present at the wire at other positions, and at the time the pulse passes by, they will all add their contributions. Calling $\varepsilon_{q}$ the field encountered by the travelling pulse, the total induced 
current $i_{a}$ will be given by

$$
i_{a}=\frac{1}{2 R_{0}} \int \varepsilon_{q} d x
$$

Since the induced current $i_{a}$ will add linearly to any current pulse already present, it is what I have previously called $\Delta i_{q}$, and we get again Eq. (B-7). 